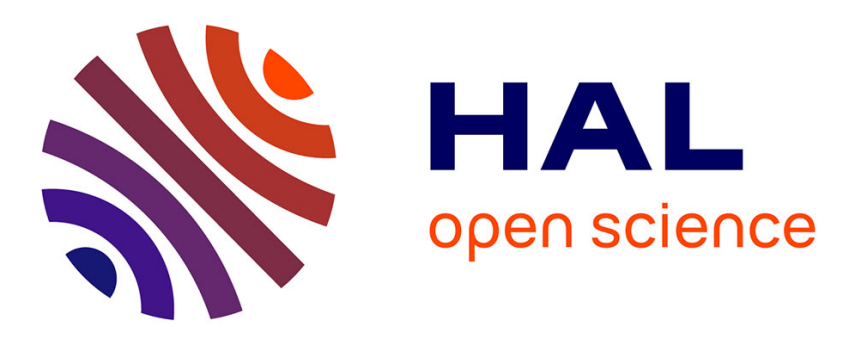

\title{
Usages des outils collaboratifs : le rôle des formes organisationnelles et des politiques de ressources humaines
}

Roxana Ologeanu-Taddei, Bernard Fallery, Ewan Oiry, Robert Tchobanian

\section{- To cite this version:}

Roxana Ologeanu-Taddei, Bernard Fallery, Ewan Oiry, Robert Tchobanian. Usages des outils collaboratifs : le rôle des formes organisationnelles et des politiques de ressources humaines. Revue management \& avenir, 2014, 67, pp.177-191. 10.3917/mav.067.0177 . hal-02049159

\section{HAL Id: hal-02049159 \\ https://hal.umontpellier.fr/hal-02049159}

Submitted on 10 Mar 2021

HAL is a multi-disciplinary open access archive for the deposit and dissemination of scientific research documents, whether they are published or not. The documents may come from teaching and research institutions in France or abroad, or from public or private research centers.
L'archive ouverte pluridisciplinaire HAL, est destinée au dépôt et à la diffusion de documents scientifiques de niveau recherche, publiés ou non, émanant des établissements d'enseignement et de recherche français ou étrangers, des laboratoires publics ou privés. 


\title{
Usages des outils collaboratifs : le rôle des formes organisationnelles et des politiques de ressources humaines
}

\author{
par Roxana Ologeanu-Tadde ${ }^{78}$, Bernard Fallery ${ }^{79}$, \\ Ewan Oiry ${ }^{80}$ et Robert Tchobanian ${ }^{81}$
}

Résumé

Nous présentons les résultats d'une enquête menée dans six organisations afin de saisir les liens entre usages des outils collaboratifs, structure organisationnelle et politique $\mathrm{RH}$. Notre recherche a mis en évidence le fait que les usages du logiciel de travail collaboratif retenu sont généralement conventionnels par rapport à des pratiques concernant des outils existants, mais qui peuvent être envisagés comme innovants par rapport aux pratiques des organisations étudiées.

Abstract

We present the results of a survey of six organizations in order to capture the relationship between use of collaborative tools, organizational structure and HR policies. Our research has shown that the use of the collaborative tool we have chosen are usually conventional compared to practices of existing tools, but can be seen as innovative in relation to the practices of the organizations we studied.

Les outils collaboratifs ont suscité l'intérêt des chercheurs depuis l'essor des technologies de groupware, dans les années 80. Cet intérêt portait sur l'observation de nouvelles pratiques de travail, qui seraient facilitées ou induites par l'implémentation de ces nouvelles technologies. II s'est manifesté notamment par des études prospectives ou normatives, concernant le design de ces technologies. En comparaison, les usages réels ont donné lieu à peu d'études empiriques. Par ailleurs, les rares études qui s'intéressent aux usages réels concluent à un certain échec dans la mesure où ces technologies ne sont pas beaucoup utilisées, et, lorsqu'elles le sont, l'usage ne réside pas toujours dans le travail collaboratif ou coopératif (Cockburn and Jones, 1995). Au-delà des discours enthousiastes des promoteurs de ces logiciels, les constats sur leur utilisation sont en demi-teinte. Beaucoup d'applications de groupware, notamment celles qui exigent la collaboration et la coopération entre utilisateurs, ne sont pas

78.Roxana Ologeanu-Taddei, Montpellier Recherche Management-CREGOR, Université Montpellier 2, roxana_ologeanu@yahoo.fr

79. Bernard Fallery, Montpellier Recherche Management, Université Montpellier 2, bernard.fallery@univ-montp 2 .fr

80. Ewan Oiry, CEREGE - IAE de Poitiers, Université de Poitiers, ewan.oiry@wanadoo.fr

81. Robert Tchobanian, LEST- UMR 6123 - Université de la Méditerranée, robert.tchobanian@univ-amu.fr 
utilisées de manière adaptée en ce sens (Orlikowski, 1993 ; Nunamaker, 1997). L'étude menée par Orlikowski (1993) sur l'utilisation de la technologie phare de groupware, Lotus Notes, dans une grande organisation, a montré par exemple que de nombreux éléments organisationnels influencent de manière significative l'implémentation et l'utilisation de cette technologie. En l'absence d'une culture de la collaboration et du partage, ce logiciel est utilisé pour des usages plus simples et classiques, tels le tableur. Lococo \& Yen (1998) vont dans le même sens, en expliquant ce constat par l'insuffisance d'une culture organisationnelle axée sur la collaboration.

Partant de là, et dans le contexte des nouveaux discours portant sur le changement organisationnel qui serait induit par les outils collaboratifs, nous nous sommes demandés si les usages de ces outils serait plus développés ou plus généralisés que les usages du groupware dans les années 1990-2000. Concrètement, nous avons souhaité répondre à la question suivante : au-delà des discours sur les bénéfices des outils collaboratifs, quelles sont les pratiques réelles de leur utilisation dans leur entreprise?

Pour répondre à cette question, nous avons essayé en premier lieu de dresser un état des lieux de la littérature, qui nous a permis de formuler quatre propositions de recherche. Nous présenterons ensuite l'enquête ${ }^{82}$ qui a permis d'explorer ces propositions, à travers six études de cas. Dans une troisième partie, nous exposerons les résultats issus de cette enquête.

\section{Revue de la littérature}

Nous nous sommes intéressés à la littérature concernant deux domaines : un domaine plus général, traitant le lien entre les types d'organisations et les usages des $\mathrm{SI} / \mathrm{TI}$, et un domaine plus spécifique portant sur l'implémentation et les usages des technologies collaboratives.

\subsection{Le rôle des caractéristiques des organisations dans les usages des $\mathrm{SI} / \mathrm{TI}$}

Dans la littérature en Systèmes d'Information (SI), l'appropriation et les usages des outils collaboratifs sont généralement abordés selon une perspective structuractionniste (Orlikowski, 1992 ; De Sanctis \& Poole, 1994). Par ailleurs, différents auteurs ont montré que les usages des SI sont liés d'une part aux caractéristiquesdelatechnologieetd'autre partauxcaractéristiquespsychologiques des utilisateurs. Peu d'auteurs ont pris en compte les caractéristiques des organisations dans l'analyse des usages. Parmi eux, Orlikowski (2000) estime

\footnotetext{
82. Notre recherche a été réalisée dans le cadre d'un contrat de recherche avec la Fondation Cigref. Cette enquête a été menée de 2010 à 2011 par une équipe de cinq enseignants-chercheurs : Bernard Fallery, Roxana Ologeanu-Taddei (CREGOR-Université Montpellier 2), Ewan Oiry, Amandine Pascal et Robert Tchobanian (LEST-Université de la Méditerranée).
} 


\section{7

que le style de management et les politiques de rémunération des salariés influencent les usages, dans la mesure où ils constituent des structures pouvant être activées dans la « technologie en pratique ».

L'importance des caractéristiques organisationnelles dans la structuration des usages a été soulignée par ailleurs dans d'autres domaines, ceux du management et de la sociologie des organisations.

Ainsi, dès 1958, Woodward affirme que les technologies déterminent directement les différences des attributs organisationnels tels la centralisation, le contrôle et la formalisation des règles et procédures. Leavitt \& Whisler (1958), George \& King (1991) et Groth (1999) se sont intéressés aux effets de la technologie sur la structure de l'organisation ; ils se sont interrogés notamment sur le rôle de la technologie sur la centralisation de l'organisation. Selon Leavitt \& Whisler (1958) par exemple, le top management peut utiliser les capacités des TI pour recentraliser les organisations. Huber (1990) et Groth (1999) estiment quant à eux que les TI peuvent aboutir à l'élimination du management intermédiaire et à une structure de l'organisation plus plate.

Des études plus récentes, ont abordé cette question des liens entre SI et organisations de manière plus nuancée, en tentant d'éviter le déterminisme technologique ou organisationnel. Nous pouvons noter ici les approches de l'adéquation (du fit) entre structure des $\mathrm{TI}$ et structure des organisations. En s'appuyant sur une analyse empirique, Fiedler et al. (1996) ont élaboré une taxonomie des structures des TI basée sur le degré de centralisation du traitement informatique, la capacité de véhiculer des communications et celle de partage de ressources. Ces auteurs ont identifié quatre structures des $\mathrm{TI}$ : centralisée (traitement informatique centralisé, communication et partage faibles), décentralisée (traitement informatique décentralisé, communication et partage faibles), centralisée coopérative (traitement informatique centralisé, communication et partage forts) et système coopératif distribué (traitement informatique décentralisé, communication et partage forts). Les TI centralisées sont liées aux organisations fonctionnelles caractérisées par une intégration faible et une prise de décision centralisée. Les TI décentralisées sont associées aux organisations où la prise de décision est décentralisée. Les systèmes coopératifs distribués sont liés aux organisations matricielles et produit avec une intégration forte (Fiedler et al., 1996).

Cependant, le lien entre structure des SI/TI, structure de l'organisation et usages effectifs (appropriation) a été très peu étudié. Ces rares études reposent sur le postulat que l'adoption d'une SI/TI va de soi, une fois que l'implémentation a été décidée par le management, car les employés seraient obligés d'utiliser la technologie. Ce lien est donc abordé dans l'optique du design organisationnel et technologique : l'essentiel serait de déterminer l'architecture organisationnelle et 
technologique des SI, puisque l'adoption ne poserait pas de problème. Or, les technologies informatiques avancées, basées sur le Web, exigent une participation croissante et volontaire de la part des utilisateurs, qui disposent d'une marge importante de liberté dans l'adoption et adaptation de la technologie. De plus, il est possible d'utiliser certaines fonctionnalités et d'en délaisser d'autres.

Plus récemment, Muhalmann (2003) par exemple a montré que le succès et l'échec d'une technologie de groupware est lié à la structure du jeu des acteurs. Selon cet auteur, ces technologies s'intègrent dans des contextes sociaux fortement interdépendants (" tightly coupled systems »), caractérisés par un fort degré de coopération, alors qu'elles sont globalement rejetées par les acteurs dans les contextes plus faiblement interdépendants (" loosely coupled systems ») et ne parviennent donc pas dans ce dernier type de configuration à modifier les jeux d'acteurs.

\subsection{Implémentation et usages des technologies collaboratives (TC)}

Plusieurs définitions des outils collaboratifs sont proposées dans la littérature. Nous avons retenu celle de Majchrzak et al. (2000), qui nous a semblé suffisamment précise : une TC, en tant que support de travail de groupe, doit comprendre un agenda, une bibliothèque de solutions et de pratiques, différentes formes d'interaction, méta-information (telle la date et le nom de l'auteur d'une contribution); elle fournit le stockage, l'accès et la transformation de l'information partagée.

Les outils collaboratifs n'échappent pas au constat que nous avons fait précédemment au sujet de l'implémentation des SI : les études qui en traitent portent sur l'implémentation des SI et sur l'architecture de la technologie (en lien avec la structure de l'organisation). Peu d'articles traitent des usages réels ; il s'agit alors d'usages dans des contextes très différents : organisation virtuelle, équipe géographiquement distribuée, étudiants, santé, fonction publique. Ces travaux portent sur des outils spécifiques : groupware, mail, visioconférence, base de connaissances, etc. Une autre différence est liée au type d'utilisateurs concernés : " travailleurs de la connaissance ", développeurs de nouveaux produits, médecins, etc.

Beaucoup d'études portent sur l'efficacité des technologies collaboratives. Or, les résultats sont contradictoires, en fonction du contexte organisationnel ou des indicateurs retenus. Le lien entre le type de tâche et l'outil collaboratif qui permet de la réaliser est une problématique dominante dans la littérature sur les usages. Les tâches peuvent être décrites en fonction de leur degré d'ambiguïté ou de complexité, de manière transversale aux différents métiers ou en fonction de leur nature liée à l'exercice d'un métier (ainsi par exemple les différentes tâches des 


\section{7

« travailleurs de la connaissance », par Kogan \& Muller, 2006). Cette approche est explorée pour les usages des outils de groupware ou de visioconférence par exemple.

Des facteurs individuels, organisationnels, technologiques et liés au groupe ont été identifiés par différents auteurs (DeSanctis \& Gallupe, 1987 ; Furst et al., 1999 ; Rice \& Grattiker, 1999 ; Sambamurthy \& Chin, 1994). Parmi ces facteurs, Majchrzak et al. (2000) se sont intéressés à l'expérience et à la tâche, celle-ci étant décrite en fonction de son degré d'ambiguïté. Par ailleurs, ils montrent que les membres de l'équipe virtuelle, qui a constitué l'objet de leur étude, utilisent le téléphone ou le face-à-face plus que la TC pour les tâches ambiguës alors ils utilisent celle-ci notamment pour les tâches ambiguës. Mais les auteurs montrent également que la TC peut être utilisée pour des tâches non ambiguës, avec une sorte d'adaptation de la tâche pour la TC (par exemple pour le brainstorming), au fur et à mesure de la création d'un langage commun. L'utilisation de la TC est moins liée à l'ambiguïté des tâches qu'à la constitution d'un langage commun et à la construction d'une expérience commune. C'est aussi ce qui peut expliquer l'augmentation de l'utilisation dans le temps.

Un autre type d'approche, plus minoritaire, cherche à trouver les liens entre les caractéristiques d'une organisation et les usages d'une TC. Pervan et al. (2005) se focalisent sur le lien entre le degré d'utilisation d'une TC, la promotion de la collaboration par le management et la structure de la prise de décision (centralisée ou décentralisée), la taille de l'organisation et celle de la fonction SI. Leurs résultats sont particulièrement intéressants pour notre recherche. Les auteurs montrent que les organisations où la collaboration est promue sont plus favorables à l'adoption d'une ou de plusieurs TC et utilisent plus les différentes fonctions d'une TC. Les organisations qui ont une structure de prise de décision plus décentralisées ont tendance à utiliser plus les différentes fonctions d'une TC. Une corrélation positive a été trouvée entre la taille de l'organisation et le degré d'utilisation d'une TC ainsi qu'entre la taille de la fonction SI et le degré d'utilisation d'une TC.

\section{Problématique et méthodologie}

L'objectif de cet article est d'approfondir ces pistes de recherche. Nous cherchions à savoir s'il existe un lien entre la structure organisationnelle (répartition des pouvoirs mais aussi mode de définition des objectifs stratégiques, en nous référant notamment à Mintzberg, 1979), l'organisation du travail et les politiques ressources humaines d'une part et les usages des technologies collaboratives d'autre part. 


\subsection{Propositions de recherche}

A partir de la revue de la littérature, nous avons élaboré quatre propositions de recherche. Celles-ci ont été validées dans la littérature dans des contextes spécifiques. Pour notre part, nous ne les avons pas envisagées comme des hypothèses au sens strict du terme, mais comme des liens entre des catégories que nous souhaitions explorer à travers des études de cas comparées.

Proposition 1. Les TC sont utilisées pour des tâches simples et ne sont pas utilisées pour des tâches ambiguës (en référence à Majchrzak et al., 2000).

Proposition 2. L'expérience, l'apprentissage et la structuration d'un langage commun permettent d'utiliser les TC pour des tâches complexes et ambiguës (Majchrzak et al., 2000).

Pour explorer ces liens, nous souhaitions mettre en évidence le type de tâches (réunion, brainstorming...) et leur objet (résoudre un problème, échanger des " trucs et astuces », rédiger une note de service, etc).

Proposition 3. Les TC sont plus utilisées dans des contextes étroitement interdépendants (en référence à Muhlmann, 2003). Les TC sont plus facilement adoptées dans des organisations décentralisées (en référence à Pervan et al., 2003).

Pour explorer ces aspects, nous avons identifé la structure et le contexte social (selon la typologie de Muhlmann) et le degré d'utilisation des TC (étendue et richesse).

Proposition 4. La promotion de la collaboration favorise la richesse des utilisations et l'adaptation de l'outil par les utilisateurs (en référence à Pervan et al., 2003). Les stratégies RH facilitatrices et les stratégies TI facilitatrices font que les TC sont plus utilisées et de manière plus variée (en référence à Tafti et al., 2007).

Nous nous sommes intéressés ici au rôle des politiques $\mathrm{RH}$ : incitation de la collaboration, formalisation de la collaboration, existence d'une fonction dédiée à l'animation etc.

\subsection{Méthodologie}

Ces propositions de recherche sont étudiées dans une démarche exploratoire, dans différentes entreprises. Pour mieux saisir le lien entre l'usage des TC et des caractéristiques organisationnelles et des politiques de ressources humaines, nous avons considéré qu'il est plus pertinent de choisir des organisations différentes qui utilisent le même logiciel et peuvent donc implémenter le même type de fonctionnalités avec les mêmes contraintes techniques. En effet, si nous avions fait varier à la fois les organisations étudiées et les technologies collaboratives analysées, nous n'aurions plus été en mesure de savoir si les 


\section{7

différences que nous identifions dans les usages doivent être attribuées aux différentes technologies collaboratives que nous étudions et/ou aux structures et politiques $\mathrm{RH}$ des entreprises analysées.

Nous avons donc retenu un logiciel, Livelink (éditeur Opentext), dont les utilisateurs ont publié des " success stories " d'utilisations collaboratives sur un site Internet dédié. Selon nous, cela constituait un élément de preuve d'utilisation du logiciel, car nous souhaitions éviter le choix d'un logiciel qui serait encore en phase d'implémentation.

A partir de ces "success stories " et de contacts donnés par les premiers utilisateurs, nous avons identifié six entreprises. Nous avons retenu la méthode des études de cas (Yin, 2002), qui nous permettrait de mieux appréhender les liens entre les différentes catégories identifiées, dans des contextes différents.

Nous avons essayé d'identifier des entreprises correspondant à des structures organisationnelles différentes (structure simple, bureaucratie, structure adhocratique, structure divisionnelle, structure matricielle). Par ailleurs, nous avons décidé, d'essayer d'analyser les usages du logiciel retenu dans deux métiers ou services de la même entreprise. Ce choix devait nous permettre de produire des résultats comparables (on se situe dans la même entreprise) tout en introduisant une certaine variété contrôlée (au sein de la même entreprise, le degré d'autonomie des employés ou les politiques RH peut différer).

Nous avons réalisé six études de cas. Pour élaborer ces études de cas, nous avons mené entre trois et six entretiens semi-directifs par cas, avec le responsable de projet d'usages collaboratifs et avec des utilisateurs. Nous avons également évalué le degré de centralisation et d'intégration de l'entreprise, selon la grille validée par Pervan et al. (2005). Cette grille permet d'évaluer, selon une échelle de Likert de 1 à 5 , le degré de centralisation d'une organisation à différents niveaux (définition du budget, décision d'introduire de nouveaux produits ou services, décision d'entrer sur de nouveaux marchés, décision des prix des principaux produits et services, définition des méthodes de sélection du personnel, définition des méthodes de travail), le degré d'intégration entre les départements (concernant les projets communs, les applications partagées, l'échange d'idées, le partage d'information, l'initiative commune de lancer de nouveaux projets).

Les entretiens ont été enregistrés et retranscrits pour faire l'objet d'une analyse de contenu manuelle, thématique. Nous avons également utilisé des données secondaires (documents d'entreprise, capture d'écran des utilisations). Pour un des cas, nous avons également réalisé une enquête par questionnaire au sein d'un service, et nous avons obtenu 42 réponses. 


\subsection{Fonctionnalités du logiciel Livelink}

Avant de présenter nos résultats, il est nécessaire de décrire brièvement le logiciel dont nous avons étudié les usages.

Livelink est un outil proposé par l'éditeur canadien Open Text, spécialisé sur les solutions de gestion de contenus d'entreprise (ECM, entreprise content management). Open Text compte 46000 clients et occupe la deuxième place sur ce marché au niveau mondial. Par ailleurs, Open Text travaille en partenariat avec SAP éditeur d'ERP, et présente aussi des solutions couplées avec Oracle.

Open Text Livelink peut être considéré comme un outil de gestion électronique des documents (GED) ; ces fonctionnalités principales ont été enrichies de fonctionnalités collaboratives :

- Gestion de documents : gestion documentaire, archivage, gestion des droits, gestion des emails, multimédia WCM...

- Gestion de processus : Workflow, processus métier (BPM), reconnaissance de documents, gestion des contenus transactionnels...

- Gestion de collaboration : contenu Web, communauté de pratique et travail collaboratif, Media sociaux, analyse sémantique, communications client, portail...

L'architecture est modulaire, ce qui permet de ne mettre en place que les fonctionnalités qu'une entreprise souhaite utiliser. Sur le poste utilisateur, Livelink ne repose que sur les technologies HTML et Javascript. Par ailleurs, les DSI des entreprises qui choisissent Livelink peuvent adapter le logiciel et l'intégrer à différentes applications Web ; les interfaces homme-machine peuvent être « customisés » différemment (par exemple par l'intégration d'un carrousel pour la navigation).

Le logiciel Livelink contient plusieurs modules qui vont de la simple classification des documents - avec une forte dominante de work-flow administratif dans ce cas - à la collaboration plus active à travers des forums, par exemple. Ce logiciel peut être utilisé pour répondre aux différents objectifs qui sont généralement visés par les technologies collaboratives (générer des connaissances, capitaliser des connaissances à travers la documentation et la codification, diffuser des connaissances par le biais d'une transmission ou d'une traduction, réutiliser des connaissances). 


\section{Résultats et discussion}

\subsection{La diversité de l'outil « unique »}

Avant de présenter les résultats relatifs à nos propositions de recherche, il convient de souligner la plasticité du logiciel. D'une part, Livelink est et peut être utilisé comme un outil de work-flow, permettant la contribution à tour de rôle sur un même document, selon des procédures de travail mis en place dans l'organisation.

Par ailleurs, Livelink peut intégrer des forums, des blogs ou être articulé des logiciels type wiki.

Le logiciel permet la « customisation », avec l'intégration d'applications Web et widgets. Cette customisation, réalisée dans la plupart des cas étudiés, aboutit à une différenciation de la technologie collaborative telle qu'elle est présentée aux utilisateurs (bien qu'il s'agisse à la base d'un même logiciel), et qui évolue de manière incrémentale au fur et à mesure des évolutions techniques ou des besoins exprimés par des utilisateurs.

\subsection{Résultats sur le lien entre usages et nature de la tâche}

Dans la majorité des cas étudiés, la TC est utilisé en tant que support pour des communautés de pratique. Celles-ci peuvent être des communautés " métier » (centrées donc sur un même métier) ou des équipes de projet, ou simplement des groupes de travail, créées par des responsables de groupes de projet (et animateurs de communauté). Dans ce cadre, Livelink est utilisé pour différentes tâches dont :

- le partage d'information (provenant de l'animateur de la communauté, qui utilise la TC pour mettre à disposition la même information à tous les membres de la communauté), dans la filiation des usages de GED,

- le travail collaboratif de type workflow

- la contribution coopérative dans le cadre de l'échange de bonnes pratiques, ce type d'usage étant impulsé et promu par le responsable des outils collaboratifs.

Si Livelink permet d'intégrer des wikis et des blogs, ces outils sont peu développés dans la plupart des cas étudiés ; et quand ils le sont, le type d'usage reste le même : la transmission et le partage d'information (par le responsable de communication par exemple ou par un animateur de communauté).

Dans une certaine mesure, les trois types de tâches présentées sont simples, non ambiguës, portant sur la transmission d'information. 
Certaines tâches qui pourraient être considérées comme étant plus ambiguës, telles la réflexion collective sur un sujet ou « lancement d'idées », peuvent être réalisées plutôt par mail ou lors de réunions. Une certaine spécialisation des outils selon les types de tâches peut être constatée : ainsi, le téléphone ou le mail sont utilisés pour poser des questions type " résolution de problèmes », pour la communication informelle ou pour le travail coopératif « bottom-up » alors que l'utilisation de Livelink reste calquée sur la transmission (et mise à disposition) de l'information, selon la logique de la GED.

Par ailleurs, le degré d'ambiguïté et de complexité de la tâche ne semble pas déterminant pour comprendre les usages. Ainsi par exemple, dans l'entreprise Alpha, des animateurs de communautés de projet se plaignent du fait que certains membres de la communauté les appellent régulièrement pour poser la même question, alors qu'il suffirait qu'ils consultent la FAQ créée sur la TC pour obtenir la réponse à la question posée. De cette manière, le téléphone ou la FAQ peuvent être utilisés pour demander une information et non pas pour réaliser une tâche de travail en tant que telle (par exemple brainstorming, prise de décision etc).

II apparaît ainsi que le critère de distinction entre les différentes tâches selon leur degré d'ambiguïté est moins pertinent que celui de la modalité de collaboration (co-construction collective, « chaîne » type workflow, partage de bonnes pratiques, partage d'information).

L'usage principal de partage d'information est résumé par un employé de la façon suivante : "si j'ai besoin d'une information j'ai un référentiel dans lequel je vais trouver cette information ". Aujourd'hui, il y a différents référentiels qui se constituent et qui, justement, permettent de ne plus dire : " tiens, est-ce que j'ai la dernière version de ce document ?». Et chercher à droite, à gauche, non. Et la collaboration, elle passe aussi par la mise à disposition de référentiels ». II s'agit donc d'un accès à une base de documents "versionnés ", qui constitue une base commune de travail. Les documents partagés sont de nature différente, selon le métier ou l'activité concernée : comptes-rendus de réunion, notes de service, documents juridiques ou comptables, états de lieux sur l'avancement du projet etc.

Pour certains utilisateurs (par exemple des architectes), les fonctions de classement et d'archivage de Livelink sont au cœur de leur activité professionnelle, ce qui explique l'importance de cette TC pour la gestion de la communauté de projet dans leur activité. 


\subsection{Résultats sur le lien entre l'expérience et l'utilisation d'une TC pour des tâches complexes et ambiguës}

Pour cet aspect, nos résultats se situent en décalage par rapport à la littérature. Puisque les usages de Livelink sont mis en place dans le cadre de communautés de projet, qui sont par nature éphémères (car liées à la durée du projet), il n'y a pas véritablement de « capitalisation » de l'expérience et un effet d'apprentissage permettant l'utilisation de la TC pour des tâches plus ambiguës.

\subsection{Résultats sur le lien entre usages de Livelink et type de structure organisationnelle}

Bien que nous ayons essayé de trouver des entreprises utilisatrices de Livelink correspondant à différentes structures organisationnelles, nous avons identifié notamment deux types de configurations : centralisée (bureaucratique) et divisionnelle. Ces deux structures sont combinées aux groupes de projets, et tendent donc à devenir des structures matricielles plus ou moins centralisées. II s'agit de grandes entreprises dont la taille et le fonctionnement (par division ou hiérarchique) rend nécessaire le classement et la gestion hiérarchisée de l'information pour un nombre importants de projets ou communautés (par exemple, dans un des cas étudiés, trois cent communautés sont mises en places, et 2100 comptes sont ouverts).

Selon la structure de l'organisation où Livelink est utilisé, son principal usage de mise à disposition de l'information selon une logique de GED peut être perçu de manière différente. Dans des organisations dont le fonctionnement est plus décentralisé et moins intégré, l'usage de ce logiciel est perçu comme un renforcement de la centralisation et le contrôle (dans deux des cas étudiés). Dans les organisations dont le fonctionnement est plutôt hiérarchique et bureaucratique, l'usage du logiciel est vu comme l' " assouplissement » du fonctionnement hiérarchique, voire comme un moyen de "court-circuiter » la hiérarchie. Si l'usage de partage d'informations peut être considéré comme étant un usage " conventionnel ", pouvant être médiatisé à travers des outils " simples ", par rapport à des usages plus innovants des technologies informatiques avancées (telle la pratique d'échange de bonnes pratiques ou de capitalisation des connaissances), cet usage est innovant dans des organisations bureaucratiques dont les employés soulignent le « culture du secret » et la rétention d'information comme source de pouvoir.

La différence de perception du logiciel Livelink selon le degré de centralisation des organisations étudiées confirme les résultats de Muhlmann (2003) que nous avons déjà présentés. 


\subsection{Résultats sur le lien entre les usages de Livelink et les politiques RH}

L'utilisation de la TC n'est prise en compte dans les objectifs de poste des employés dans aucun des cas étudiés. C'est plutôt l'animation de la communauté de projet qui est intégrée dans les objectifs lors des entretiens individuels annuels.

Dans quatre cas étudiés, la politique $\mathrm{RH}$ ne favorise pas le travail collaboratif en tant que tel mais le management a créé une fonction de responsable des outils collaboratifs qui promeut les fonctionnalités et les utilisations potentielles de ces technologies. Or, le rôle du responsable d'outils collaboratifs, qui est un cadre informatique, est celui du support fonctionnel : il propose et met à disposition des outils mais n'a pas de pouvoir de prise de décision. Les objectifs de poste et les tâches étant généralement déterminés par les managers directs, si ceux-ci ne promeuvent pas l'utilisation des TC et ne « donnent pas l'exemple » en les utilisant (comme cela est le cas dans la majorité des cas étudiés), les employés estiment que l'utilité de ces TC est limitée. En l'absence d'une politique de promotion de la collaboration (Pervan et al., 2005), la TC induit pourtant, par son usage innovant (pour l'organisation) de partage d'information, un certain changement allant vers une culture de la collaboration, embryonnaire. Néanmoins, le logiciel est peu utilisé, et reste un outil parmi d'autres dans un éventail d'autres outils disponibles dont les plus utilisés demeurent le téléphone et le mail.

Il est important de noter que nous n'avons relevé dans aucun des cas étudiés des pratiques $\mathrm{RH}$ facilitatrices des pratiques d'utilisation des technologies collaboratives telles la valorisation de l'autonomie des employés, leur implication dans plusieurs équipes de résolution de problèmes (sauf si l'on considère comme telle une équipe projet), la flexibilité du poste de travail, exigeant une rotation entre différentes tâches, partage régulier de l'information entre les managers et les employés, les premiers sollicitant l'opinion des derniers (Tafti et al., 2007). Bien au contraire, les pratiques RH sont plutôt traditionnelles (Tafti et al., 2007), focalisées sur le contrôle managérial et par les procédures ainsi que sur la définition précise des postes et sur l'absence d'une culture de partage de l'information entre managers et employés.

\subsection{Des résultats émergents}

Au-delà des résultats correspondant à nos propositions initiales, d'autres constats se dégagent de nos études de cas.

Le premier porte sur la tension existante entre la logique de collaboration professionnelle et la logique de la sécurité informatique portée par les DSI. En effet, Livelink est peu utilisé lorsque des employés (tels par exemple des experts d'un domaine comme la recherche et l'innovation) ont besoin de travailler 


\section{7

avec des professionnels (au sens de Mintzberg, 1979) qui exercent le même métier qu'eux, dans le cadre par exemple de réseaux professionnels formalisés, permettant la création de savoirs communs, mobilisés dans l'entreprise. Dans cette situation (que nous avons rencontrée dans deux cas étudiés), les cadres concernés sont très critiques à l'égard de Livelink et des systèmes d'information implémentés dans l'entreprise en général, et préfèrent s'en détourner au profit de logiciels grand public existant sur le Web. Tel est par exemple le cas d'un logiciel comme Skype, plébiscité pour sa facilité d'utilisation et son utilisation par des collaborateurs externes, dont l'utilisation en entreprise est pourtant freinée par les DSI qui mettent en avant l'exigence de la sécurité informatique.

Un second constat concerne la disponibilité et l'utilisation de la TC dans le cadre d'un éventail d'autres outils informatiques, dont certains sont concurrents. Ainsi par exemple dans un des cas étudiés, les employés ont accès à la TC à travers un portail personnalisé, qui agrège par ailleurs l'information de différentes applications ; ils peuvent consulter également trois intranets (celui de l'entreprise, celui de la branche d'activité et celui du service) et utiliser des logiciels plus ou moins concurrents, permettant de faire la même tâche. Concernant Livelink par exemple, son utilisation peut être similaire à l'utilisation d'un logiciel spécialisé par métier (qui n'est pas étiqueté comme étant collaboratif). L'arbitrage entre l'un ou l'autre est réalisé par l'employé, en fonction de ses habitudes et de celles des employés avec lesquels il travaille. Par conséquent, l'intensité d'utilisation et la variété d'utilisation d'un logiciel tel Livelink devrait être analysées en lien avec l'intensité d'utilisation et la variété d'utilisation d'autres logiciels qui permettent de réaliser des tâches similaires.

\section{Conclusion}

Dans notre recherche exploratoire, nous avons tenté de saisir le lien existant entre le choix d'un outil collaboratif, les usages qui en sont fait et la structure organisationnelle. Dans le cas de la technologie que nous avons retenue, les fonctionnalités collaboratives se greffent sur les fonctionnalités de gestion électronique des documents, qui restent les plus utilisés. Cependant, dans la plupart des cas étudiés, la simple mise à disposition de l'information dans le cadre d'une communauté de projet est définie par les employés comme un usage collaboratif de la technologie (dans le sens de mise en commun et de partage de l'information).

Dans les six organisations étudiées, il n'existe pas de pratiques RH qui facilitent les usages des outils collaboratifs, ou le travail collaboratif médiatisé par ces outils. L'existence d'une fonction de responsable des outils collaboratifs permet de faire la promotion et de l'accompagnement des utilisateurs mais n'est pas suffisante pour permettre l'essor d'autres types d'usages collaboratifs ou la généralisation des pratiques de partage d'information. 


\section{Bibliographie}

BOUNFOUR A. (2009), Organisational capital, Modelling, Measuring, Contextualising, Routledge, Londres.

BERGERON F., RAYMOND L., RIVARD S. (2001), « Fit in strategic information technology management research: an empirical comparison of perspectives ", Omega - The International Journal of Management Science, Vol. 29, p. 125-142.

BROWN C.-V., MAGILL S.-L. (1994), « Alignment of the IS function with the enterprise: toward a model of antecedents », MIS Quarterly, Vol. 18, No. 4, p. 371-403.

BURNS T., STALKER G.-M. (1961), The Management of Innovation, Tavistock Publications, Londres.

COCKBURN A. \& JONES S. (1995), « Four principles of groupware design », Interacting with Computers, Vol. 7, No. 2, p. 195-210.

DESANCTIS G., GALLUPE B. (1987), "A foundation for the study of group decision support systems", Management Science, Vol. 33, No.5, p. 589-609.

DE SANCTIS G. \& POOLE M.-S. (1994), "Capturing the Complexity in Advanced Technology Use: Adaptative Structuration Theory», Organization Science, Vol. 5, No.2, p. 121-147.

FIEDLER KD, GROVER V, TENG J.-T.-C. (1996), "An empirically derived taxonomy of information technology structure and its relationship to organizational structure", Journal of Management Information Systems, Vol.13, No.1, p.9-34.

FURST S., BLACKBURN R., ROSEN B. (1999), "Virtual teams: a proposed research agenda", Information Systems Journal, Vol. 9 p. 249-69.

GEORGE J.-F. \& KING J.-L. (1991), "Examining the computing and centralization debate", Communications of the ACM, Vol. 34, p. $62-72$.

GROTH L. (1999), Future organizational design: the scope for the IT-based enterprise, Easthbourne, John Wiley \& Sons.

HUBER G.-P. (1990), "A theory of the effects of advanced information technologies on organizational design, intelligence, and decision making", Academy of Management Review, Vol. 15, No. 1, p. 47-71.

LEAVITT H. \& WHISLER T. (1958), "Management in the 1980's", Harvard Business Review, November-December, p. 41-48.

LOCOCO A. \& YEN D.-C. (1998), "Groupware: computer supported collaboration", Telematics and Informatics, Vol. 15, p. 85-101.

MAJCHRZAKA., RICE R., KING N., MALHOTRAA., BAS. (2000), "Technology adaptation: The case of a computer-supported inter-organizational virtual team", MIS Quarterly, Vol. 24, No.4, p. 569-600.

MINTZBERG H. (1979), The structuring of organizations: a synthesis of the research, Englewood Cliffs, N.J., Prentice Hall.

MUHLMANN D. (2003), L'impact organisationnel des nouvelles technologies. Le cas du groupware et du knowledge management, Thèse de doctorat, IEP, Paris.

NUNAMAKER J.-F., O BRIGGS R., MITTLEMAN D.-D., VOGEL D.-R., BALTHAZARD P. (1997), "Lessons from a dozen years of group support systems research: A discussion of Lab and Field findings", Journal of Management Information Systems, Vol.13, No.3, p. 163-207.

Orlikowski W. (1993), "Learning from notes: Organizational issues in groupware implementation", The Information Society, Vol. 9, No. 3, p. 237-250.

ORLIKOWSKI W. (1992), "The Duality of Technology: Rethinking the Concept of Technology in Organizations”, Organization Science, Vol. 3, No. 3, p. 398-427.

RICE R.-E., GATTIKER U. (1999), "New media and organizational structuring of meanings and relations". In F. Jablin and L. Putnam (eds.), New handbook of organizational communication, Newbury Park, Sage. 


\section{7 \\ management \& avenir}

SAMBAMURTHY V., CHIN W. (1994), "The effects of group attitudes toward alternative GDSS designs on the decision-making performance of computer-supported groups", Decision Sciences, Vol. 25, No. 2, p. 215-241.

TAFTI A., MITHAS S., KRISHNAN M.-S. (2007), "Information technology and the autonomy-control duality: toward a theory", Information Technology Management, Vol. 8, p. 147-166.

WOODWARD J. (1958), Management and technology, Department of Scientific and Industrial Research; Problems of Progress on Industry, Her Majesty's Stationery Office, Londres.

YIN R.-K. (2002), Case Study Research. Design and Methods. Third Edition. Applied social research method series, Sage Publications, California. 